\title{
REGISTRO DE UN DINOSAURIO AEOLOSAURINI (SAUROPODA, TITANOSAURIA) EN EL CRETÁCICO SUPERIOR (FORMACIÓN PLOTTIER) DEL NORTE DE LA PROVINCIA DE NEUQUÉN, ARGENTINA, Y COMENTARIOS SOBRE LOS AEOLOSAURINI SUDAMERICANOS
}

\author{
LEONARDO S. FILIPPI \\ Museo Municipal Argentino Urquiza, Jujuy y Chaco s/n, (Q8319BFA), Rincón de los Sauces, \\ Neuquén, Argentina.lsfilippi@yahoo.com.ar \\ AGUSTÍN G. MARTINELLI \\ Centro de Pesquisas Paleontológicas L.I. Price, Complexo Cultural e Científico Peirópolis, UFTM, \\ BR-262, km 784, Peirópolis, 38001-970, Uberaba, MG, Brasil.agustin_martinelli@yahoo.com.ar \\ ALBERTO C. GARRIDO \\ Museo Provincial de Ciencias Naturales "Prof. Dr. Juan Olsacher”, Dirección Provincial de Minería, \\ Etcheluz y Ejército Argentino, (Q8340AUD) Zapala, Neuquén, Argentina. algene@copelnet.com.ar
}

\begin{abstract}
RECORD OF AN AEOLOSAURINI DINOSAUR (SAUROPODA, TITANOSAURIA) IN THE UPPER CRETACEOUS (PLOTTIER FORMATION) OF NORTHERN NEUQUÉN PROVINCE, ARGENTINA, AND COMMENTS ON THE SOUTH AMERICAN AEOLOSAURINI. The clade Aeolosaurini is represented by several specimens found, so far, only in Argentina and Brazil. The material reported here corresponds to a sauropod titanosaur consisting of four incomplete anterior caudal vertebrae, from the Narambuena Paleontological Site, Rincón de los Sauces, Neuquén Province, Argentina. The specimen comes from the Plottier Formation (late Coniacian-lower Santonian, Upper Cretaceous), Neuquén Group. The specimen has a combination of features that includes it into the clade Aeolosaurini: anterior caudal centra with anterodorsal margin bent forward; prezygapophyses elongated in anterior caudal, in correlation with the extreme displacement of the neural arch forward; and anteroposteriorly elongated articular facets of prezygapophyses, at least in the anterior caudals. However, it differs from other Aeolosaurini by having postzygapophysis with anteroposteriorly short articular facets, not as elongated in the prezygapophyses. This specimen corresponds not only to the first discovery of an Aeolosaurini in the north of the Neuquén Basin (because Rinconsaurus caudamirus Calvo \& Gonzalez Riga has been included in another clade, Rinconsauria), but also the oldest record of the group.
\end{abstract}

Key words: Dinosauria, Aeolosaurini, Plottier Formation, late Coniacian-lower Santonian, Upper Cretaceous, Neuquén Group.

RESUMO - O clado Aeolosaurini é representado por diversos espécimes encontrados, até o momento, somente na Argentina e Brasil. O material dado a conhecer pertence a um saurópodo titanossauro e corresponde a quatro vértebras caudais anteriores incompletas provenientes do sítio paleontológico Narambuena, Rincón de los Sauces, Provincia de Neuquén, Argentina. O material é proveniente da Formação Plottier (Coniaciano tardio/Santoniano inferior, Cretáceo Superior), Grupo Neuquén. As vértebras possuem uma série de caracteres que permitem inclúi-las no clado Aeolosaurini: (i) corpos anteriores com a margen anterodorsal inclinada anteriormente; (ii) pré-zigapófises alongadas nas caudais anteriores, em correlação com o extremo deslocamento do arco neural para frente; e (iii) facetas articulares das pré-zigapófises alongadas anteroposteriormente, ao menos nas caudais anteriores. Não obstante, se diferenciam das de outros Aeolosaurini por apresentar pós-zigapófises com facetas articulares não alongadas anteroposteriormente, como ocorre com as facetas das pré-zigapófises. Estes materiais correspondem não apenas ao primeiro achado de um Aeolosaurini para o norte da bacia Neuquina (devido a que Rinconsaurus caudamirus Calvo \& Gonzalez Riga foi incluído em outro clado, Rinconsauria), como também ao registro mais antigo do grupo.

Palavras-chave: Dinosauria, Aeolosaurini, Formação Plottier, Coniaciano tardio-Santoniano inferior, Cretáceo Superior, Grupo Neuquén.

\section{INTRODUCCIÓN}

El registro de titanosaurios Aeolosaurini (Sauropoda, Titanosauria) consiste en diversos especímenes hallados hasta el momento sólo en Argentina y Brasil. De acuerdo a la propuesta filogenética de Santucci \& ArrudaCampos (2011), en el clado Aeolosaurini se incluirían las siguientes especies: Maxakalisaurus topai Kellner et al., 
2006, Gondwanatitan faustoi Kellner \& Azevedo, 1999 y Aeolosaurus maximus Santucci \& Arruda-Campos, 2011, provenientes de Brasil (Kellner \& Azevedo, 1999; Kellner et al., 2006; Santucci \& Arruda-Campos, 2011), y Aeolosaurus rionegrinus Powell, 1987, A. colhuehuapensis Casal et al., 2007, Panamericansaurus schroederi Calvo \& Porfiri, 2010 y Rinconsaurus caudamirus Calvo \& González Riga, 2003, provenientes de Argentina (Powell, 1987, 2003; Casal et al., 2007; Calvo \& González Riga, 2003). Asimismo, a este grupo se asignan otros registros basados en restos aislados del Cretácico Superior de Brasil (véase Martinelli et al., 2011) y Argentina (e.g. Salgado \& Coria, 1993; Salgado et al., 1997; García \& Salgado, en prensa). Particularmente, los registros argentinos de Aeolosaurini son los siguientes: (i) Aeolosaurus rionegrinus, de la localidad Casa de Piedra, Río Negro, Formación Angostura Colorada (posiblemente Campaniano tardío) (Powell, 1987, 2003); (ii) Aeolosaurus sp., de la localidad Salitral Moreno, Río Negro, Formación Allen (Campaniano-Maastrichtiano) (Salgado \& Coria, 1993; García \& Salgado, en prensa); (iii) Aeolosaurus sp., de la localidad Los Alamitos, Río Negro, Formación Los Alamitos (Campaniano-Maastrichtiano) (Salgado et al., 1997); (iv) A. colhuehuapensis, del Lago Colhué Huapi, Chubut, Formación Bajo Barreal (?Campaniano-Maastrichtiano) (Casal et al., 2007); y (v) Panamericansaurus schroederi, hallado en las cercanías de San Patricio del Chañar, Neuquén, Formación Allen (Campaniano-Maastrichtiano) (Calvo \& Porfiri, 2010). Recientemente, el material de Aeolosaurus sp. proveniente de Salitral Moreno fue referido a la especie P. schroederi, taxón que sería propio de la Formación Allen (Juárez Valieri \& Bellardini, 2012). Asimismo, Juárez Valieri \& Bellardini (2012) plantean dudas sobre la validez del género Panamericansaurus Calvo \& Porfiri, 2010 (pero no de la especie), por lo que sugieren que este género podría ser un sinónimo de Aeolosaurus Powell, 1987. Rincosaurus caudamirus proveniente de Cañadón Río Seco (Calvo \& González Riga, 2003), Rincón de los Sauces, Neuquén, Formación Anacleto (Garrido, 2010), también ha sido relacionado con los Aeolosaurini (e.g. Calvo \& González Riga, 2003; Santucci \& Arruda-Campos, 2011), sin embargo, el análisis de Calvo et al. (2007) posiciona a Rinconsaurus Calvo \& González Riga, 2003 junto a Muyelensaurus pecheni Calvo, Gonzalez-Riga \& Porfiri, 2007 en el clado Rinconsauria.

En el presente trabajo se dan a conocer los restos de un saurópodo titanosaurio provenientes del Yacimiento Narambuena, Rincón de los Sauces, Provincia de Neuquén, Argentina (Figura 1), cuyos niveles portadores son asignados a la Formación Plottier, Grupo Neuquén, Cretácico Superior (Santoniano inferior; Garrido, 2010). De acuerdo a los caracteres observados (véase discusión) en el espécimen (i.e. una secuencia de cuatro vértebras caudales incompletas), y siguiendo la propuesta de Franco-Rosas et al. (2004), este nuevo ejemplar es considerado como un miembro del clado Aeolosaurini, constituyendo un nuevo registro para la Argentina y el registro más antiguo de este grupo.

\section{MARCO GEOLÓGICO}

El material de estudio ha sido recuperado en niveles de fangolitas rojizas pertenecientes a la Formación Plottier, con una edad estimada entre el Coniaciano tardío y el Santoniano temprano (Cretácico Superior; Garrido, 2010). Esta unidad integra la secuencia fluvial del Cretácico Superior de la Cuenca Neuquina reconocida bajo la denominación de Grupo Neuquén, conformando los niveles superiores del denominado Subgrupo Río Neuquén (Ramos, 1981; Garrido, 2010).

En el área del hallazgo la Formación Plottier exhibe 95 $\mathrm{m}$ de espesor (Figura 2), encontrándose caracterizada por el desarrollo de una sucesión de dominio pelítico de coloración rojiza, con intercalaciones de cuerpos canalizados de moderada envergadura compuestos de areniscas cuarcíticas de granulometría media y coloración gris-blanquecina. El nivel fosilífero corresponde a facies de fangolitas de aspecto macizo a tenuemente laminadas, correspondientes a depósitos de planicie de inundación asociados a sistemas fluviales de baja energía (Garrido, 2010). Dentro de la sucesión, los restos fósiles se ubican sobre el tramo superior de la unidad, a aproximadamente $11 \mathrm{~m}$ del contacto con la Formación Bajo de la Carpa (Figura 2).

Abreviaturas institucionales. CPPLIP, Centro de Pesquisas Paleontológicas Llewellyn Ivor Price, Peirópolis, Uberaba, MG, Brasil; LGP-D, Laboratório de Geologia e Paleontologia, Fundação Universidade Federal do Rio Grande, Rio Grande, RS, Brasil; MAU-Pv, Paleontología de Vertebrados, Museo Municipal "Argentino Urquiza", Rincón de los Sauces, Neuquén, Argentina; MP, Museu de Paleontologia da Universidade Federal do Mato Grosso, MT, Brasil; MPCA, Museo Provincial "Carlos Ameghino", Cipolletti, Río Negro, Argentina; MPMA, Museu de Paleontologia "Prof. Antonio Celso de Arruda Campos", Monte Alto, SP, Brasil.

\section{SISTEMÁTICA PALEONTOLÓGICA}

\author{
Superorden DINOSAURIA Owen, 1842 \\ Orden SAURISCHIA Seeley, 1888 \\ Infraorden SAUROPODA Marsh, 1878 \\ (Clado sin rango) TITANOSAURIA Bonaparte \\ \& Coria, 1993 \\ Tribu AEOLOSAURINI Franco-Rosas, Salgado, \\ Rosas \& Carvalho, 2004
}

Gen. et sp. indet.

(Figuras 3-5, Tabla 1)

Materiales. MAU-Pv-N-414, secuencia articulada de cuatro vértebras caudales anteriores y fragmentos de arco neural caudal.

Localidad. Yacimiento Narambuena, $25 \mathrm{~km}$ al oeste de la localidad de Rincón de los Sauces, Neuquén, Argentina.

Horizonte y edad. Formación Plottier, Subgrupo Río Neuquén, Grupo Neuquén; Coniaciano tardío/ Santoniano temprano, Cretácico Superior (Garrido, 2010). 


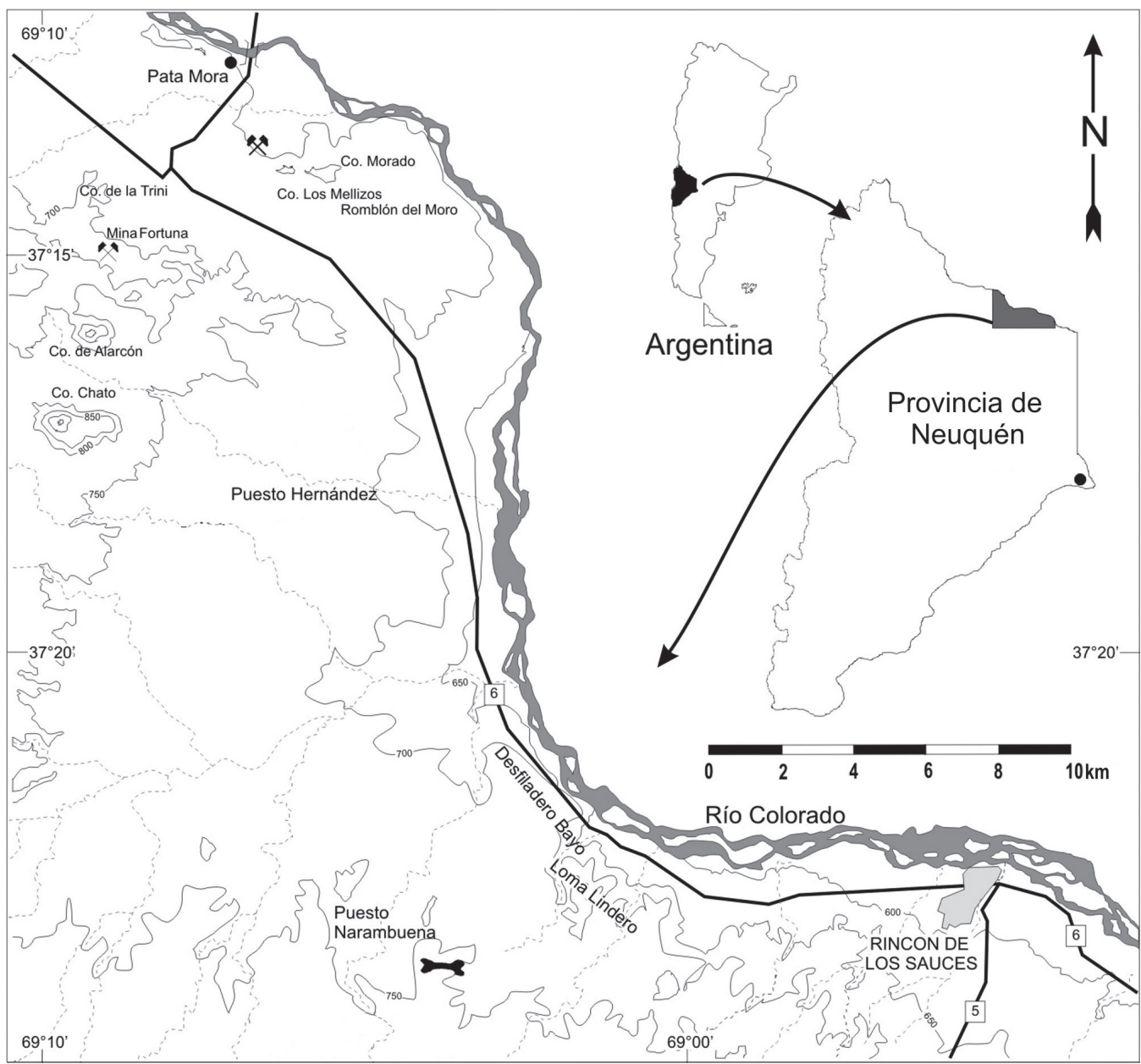

Figura 1. Mapa de ubicación del yacimiento Narambuena, Rincón de los Sauces, Provincia de Neuquén, Argentina, donde fue hallado el ejemplar MAU-Pv-N-414.

Figure 1. Location map of the Narambuena site, Rincón de los Sauces, Neuquén Province, Argentina, where the specimen MAU-Pv-N-414 was found.

\section{DESCRIPCION Y COMPARACIONES}

El material corresponde a una secuencia de cuatro vértebras caudales (Figura 3 ) y fragmentos de un arco neural caudal, que probablemente corresponda a alguna de las dos primeras vértebras incompletas de la secuencia. De acuerdo con comparaciones con otros titanosaurios como Aeolosaurus colhuehuapensis, A. maximus, Baurutitan britoi Kellner, Campos \& Trotta, 2005) y Trigonosaurus pricei Campos et al., 2005, dichas vértebras son interpretadas como caudales (i.e. Ca), probablemente $\mathrm{Ca} 4, \mathrm{Ca} 5, \mathrm{Ca} 6$ y Ca 7. Si bien la preservación del material es buena, la primera de la serie (Ca 4) sólo ha preservado parcialmente el cuerpo vertebral, y la segunda ( $\mathrm{Ca} 5$ ) el cuerpo vertebral con su borde lateral izquierdo incompleto, observándose en vista dorsal restos de la base del arco neural. La tercera vértebra (Ca 6) (Figura 4) es la más completa de la secuencia. Sin embargo, la carilla articular de la prezigapófisis derecha, los procesos transversos y la porción distal de la espina neural no están preservados en esta vértebra. La cuarta vértebra (Ca 7) (Figura 5), carece del proceso transverso izquierdo, parte del derecho y de la espina neural.
Todas las vértebras son fuertemente procélicas, con el cótilo proporcionalmente más ancho que el cóndilo, lo que se observa principalmente en la Ca 5 . El ápice del cóndilo no se encuentra posicionado en forma central como ocurre en Aeolosaurus colhuehuapensis, sino que se ubica en una posición más dorsal, como en A. maximus. Si bien las vértebras presentan cuerpos lateralmente comprimidos, en la $\mathrm{Ca} 4$ y Ca 5 , éstos son ventralmente más anchos. Las caras laterales son prácticamente planas dorsoventralmente, sobre todo en la $\mathrm{Ca}$ 6, y anteroposteriormente cóncavas. Hacia la Ca 7, el cuerpo vertebral se hace más alto que ancho y la cara ventral es relativamente más estrecha, como ocurre en las caudales de $A$. rionegrinus. La cara ventral es anteroposteriormente cóncava y prácticamente plana lateralmente, exhibiendo facetas articulares para las hemapófisis bien desarrolladas. El arco neural se encuentra ubicado en el borde anterior del cuerpo vertebral, con las prezigapófisis proyectadas anterodorsalmente y con el extremo articular orientado anteriormente, al igual que en Aeolosaurus sp. y A. rionegrinus (Salgado et al., 1997; Powell, 2003). El borde anterior del cuerpo vertebral se encuentra ligeramente inclinado hacia adelante. Las prezigapófisis son relativamente largas, iguales o ligeramente más largas que 


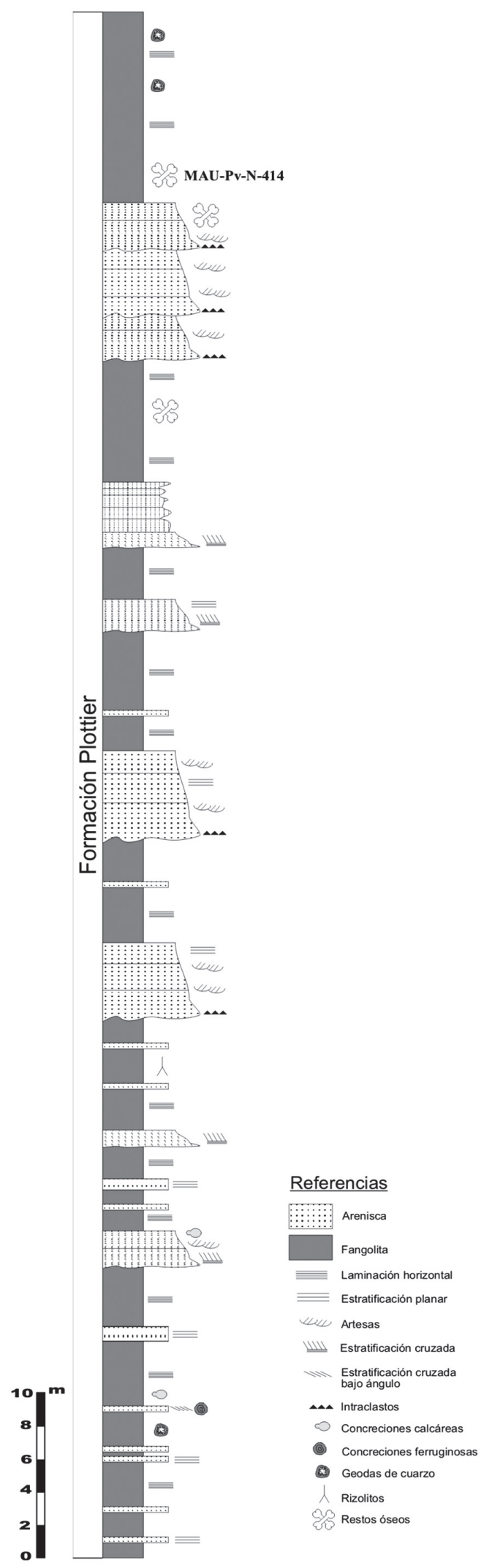

Figura 2. Sección litoestratigráfica de la Formación Plottier (Subgrupo Río Neuquén, Grupo Neuquén) en el área de Puesto Narambuena, Rincón de los Sauces, Provincia de Neuquén, Argentina.

Figure 2. Litoestratigraphic section of the Plottier Formation (Neuquén Subgroup, Neuquén Group) in the Narambuena area, Rincón de los Sauces, Neuquén Province, Argentina. la longitud del cuerpo vertebral. Las prezigapófisis se hallan unidas a la espina neural por láminas espinoprezigapofiseales bien definidas, las cuales delimitan una fosa prespinal junto al techo del canal neural (Figura 4). Las carillas articulares de las prezigapófisis, de contorno suboval, poseen un diámetro anteroposterior ligeramente mayor que el diámetro dorsoventral. En cambio, las postzigapófisis son de contorno subtriangular, con carillas articulares de diámetro dorsoventral mayor que el anteroposterior, y posicionadas en la mitad del cuerpo vertebral, como ocurre en Panamericansaurus schroederi. En Aeolosaurus rionegrinus y A. cohuelhuapensis, en cambio, las postzigapófisis se encuentran posicionadas a la altura del borde anterior del cuerpo vertebral. Las postzigapófisis están unidas a la espina neural por medio de una lámina espinopostzigapofiseal que delimita una fosa postespinal, la cual está dividida por una lámina postespinal (Figura 4). Si bien la espina neural no se ha preservado completa en las vértebras recuperadas, en la $\mathrm{Ca} 6$, la cual ha preservado su porción proximal, se infiere que ésta se inclinaba levemente hacia atrás o en una posición bastante vertical y no anteriormente como en Aeolosaurus sp. (MPCA-27100) (Salgado et al., 1997), A. rionegrinus y $P$. schroederi, donde en las caudales anteriores la espina se encuentra inclinada hacia adelante, situando su extremo a la altura del borde anterior del cuerpo vertebral.

\section{DISCUSION}

Taxonomía de MAU-Pv-N-414 y Aeolosaurini patagónicos

Las rocas del Cretácico Superior en el área de Rincón de los Sauces (Provincia de Neuquén), ha proporcionado una notable diversidad de dinosaurios saurópodos titanosaurios, como el ejemplar MAU-Pv-AC-01 (Calvo et al., 1997), Rinconsaurus caudamirus, Muyelensaurus pecheni, Pitekunsaurus macayai Filippi \& Garrido, 2008, Narambuenatitan palomoi Filippi, García \& Garrido 2011 a y Petrobrasaurus puestohernandezi Filippi et al., 2011b. De estos taxones, M. pecheni y $P$. puestohernandezi provienen de la base de la Formación Plottier, R. caudamirus de la Formación Bajo de la Carpa, y $P$. macayai, $N$. palomoi y el ejemplar MAU-Pv-AC-01 provienen de la Formación Anacleto (Garrido, 2010). A esta lista se suma el ejemplar MAU-Pv-N-414 (Figura 3) aquí descripto que proviene de la sección superior de la Formación Plottier.

MAU-Pv-N-414 exhibe una serie de caracteres que lo posicionan como un miembro del grupo Aeolosaurini, definido por Franco-Rosas et al. (2004) como el clado más inclusivo que contiene a Aeolosaurus rionegrinus y Gondwanatitan faustoi, pero no a Saltasaurus loricatus Bonaparte \& Powell, 1980 ni a Opisthocoelicaudia skarzynskii Borsuk-Bialynicka, 1977. De los caracteres usados por Franco-Rosas et al. (2004) para diagnosticar este clado ( Campos, 2011, p. 5), el espécimen aquí presentado posee los siguientes: cuerpos caudales anteriores con el margen anterior del cuerpo vertebral inclinado hacia adelante; prezigapófisis alargadas en las caudales anteriores, en correlación con el desplazamiento del arco neural hacia adelante; facetas articulares de las prezigapófisis alargadas anteroposteriormente, al menos en las caudales anteriores. A diferencia de otros taxones 


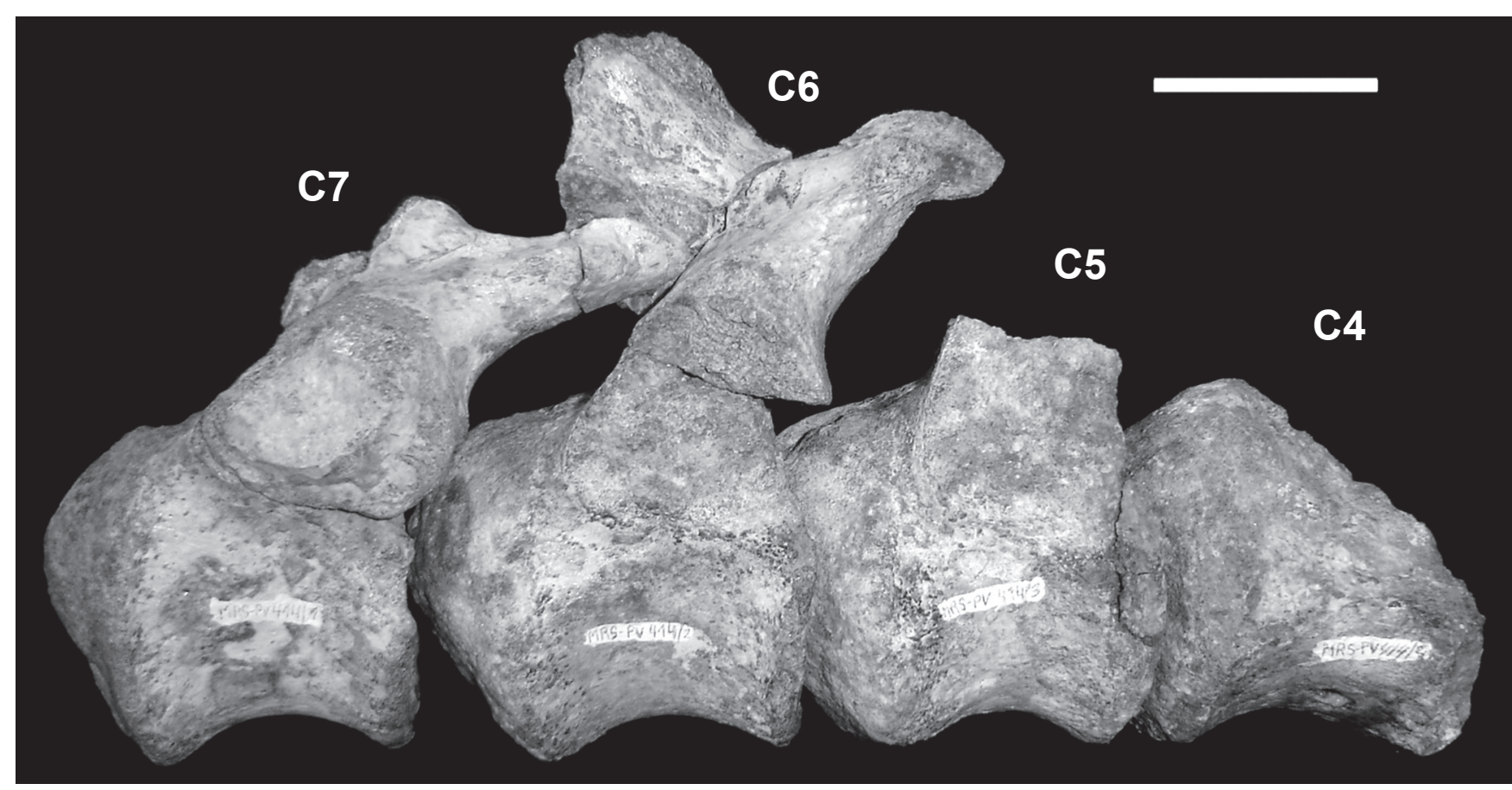

Figura 3. Aeolosaurini indet. MAU-Pv-N-414, Vista lateral derecha de la secuencia de vertebras caudales. Abreviaturas: C4-C7, caudales 4 a 7 . Escala $=5 \mathrm{~cm}$.

Figure 3. Aeolosaurini indet. MAU-Pv-N-414, right lateral view of the caudal series. Abbreviations: $\mathrm{C} 4-\mathrm{C} 7$, caudals 4 a $7 . \mathrm{Scale}$ bar $=5 \mathrm{~cm}$.

de Aeolosaurini, MAU-Pv-N-414 presenta postzigapófisis con facetas articulares no alargadas anteroposteriormente, a diferencia de lo que ocurre con las facetas de las prezigapófisis. Debido a la naturaleza fragmentaria del material y a la imposibilidad, en el presente estudio, de evaluar este carácter en un marco filogenético, no podemos afirmar si esta condición es plesiomórfica para este Aeolosaurini.

En relación a los titanosaurios hallados en localidades cercanas a Rincón de los Sauces, el nuevo ejemplar MAU$\mathrm{Pv}-\mathrm{N}-414$ presenta significativas diferencias. Los centros caudales son más cortos, más anchos y más altos que en el ejemplar MAU-Pv-AC-01 (Calvo et al., 1997), Rinconsaurus, Pitekunsaurus Filippi \& Garrido, 2008 y Narambuenatitan Filippi, García \& Garrido 2011. En Pitekunsaurus como en Petrobrasaurus Filippi et al., 2011, el arco neural se ubica apenas sobre la mitad anterior del cuerpo vertebral, mientras que en el ejemplar MAU-Pv-AC-01, como en Narambuenatitan lo hace dorsoanteriormente; pero en MAU$\mathrm{Pv}-\mathrm{N}-414$ como en Muyelensaurus Calvo, Gonzalez-Riga \& Porfiri, 2007, al menos en una de las caudales más anteriores recuperadas (Calvo et al., 2007, fig. 9), se ubica sobre el borde anterior del centro. En el ejemplar MAU-Pv-N-414, tanto el arco neural como las prezigapófisis son robustas y se proyectan anterodorsalmente. La caudal anterior de Pitekunsaurus posee un arco neural bajo con prezigapófisis gráciles que se proyectan ligeramente hacia adelante. En el ejemplar MAU-Pv-AC-01, en Petrobrasaurus y en Narambuenatitan el arco neural es bajo, pero de constitución robusta al igual que las prezigapófisis. Las carillas articulares de las prezigapófisis en Petrobrasaurus y Narambuenatitan difieren de las del nuevo ejemplar, ya que son dorsoventralmente amplias, sobre todo en las caudales más anteriores de Narambuenatitan. Aunque en el ejemplar MAU-Pv-N-414, de las espinas neurales sólo se halla preservada la región proximal en la C6, ésta difiere de la presente en Rinconsaurus y Pitekunsaurus, la cual es anteroposteriormente alargada y transversalmente comprimida, adquiriendo un aspecto laminar. En Narambuenatitan como en el ejemplar MAUPv-AC-01, la morfología de las espinas neurales varía en la secuencia caudal. En Narambuenatitan dicha variación se da desde la primera caudal, la cual es transversalmente ancha, pasando a ser cuadrangular en la segunda y tercera caudal, de modo similar a lo observado en el ejemplar MAU$\mathrm{Pv}-\mathrm{N}-414$, hasta finalmente adquirir un aspecto laminar en las últimas caudales anteriores. En el ejemplar MAU-Pv-AC-01, la espina neural de las primeras caudales es más extendida anteroposteriormente que lateralmente, mientras que en las caudales más posteriores, la espina neural se encuentra comprimida lateralmente. En Petrobrasaurus como en la caudal más anterior recuperada de Muyelensaurus de acuerdo con Calvo et al. (2007, fig. 9) la espina neural es robusta y de sección cuadrangular, encontrándose ligeramente inclinada posteriormente.

La presencia en el ejemplar MAU-Pv-N-414 de prezigapófisis relativamente más cortas que en las especies de Aeolosaurus de Patagonia y la menor inclinación de la espina neural son caracteres también compartidos con Panamericansaurus schroederi proveniente de la Formación Allen de la localidad de San Patricio del Chañar, Neuquén (Calvo \& Porfiri, 2010). Como un análisis filogenético está fuera del objetivo del presente trabajo, la importancia de estos caracteres es aún controversial. 


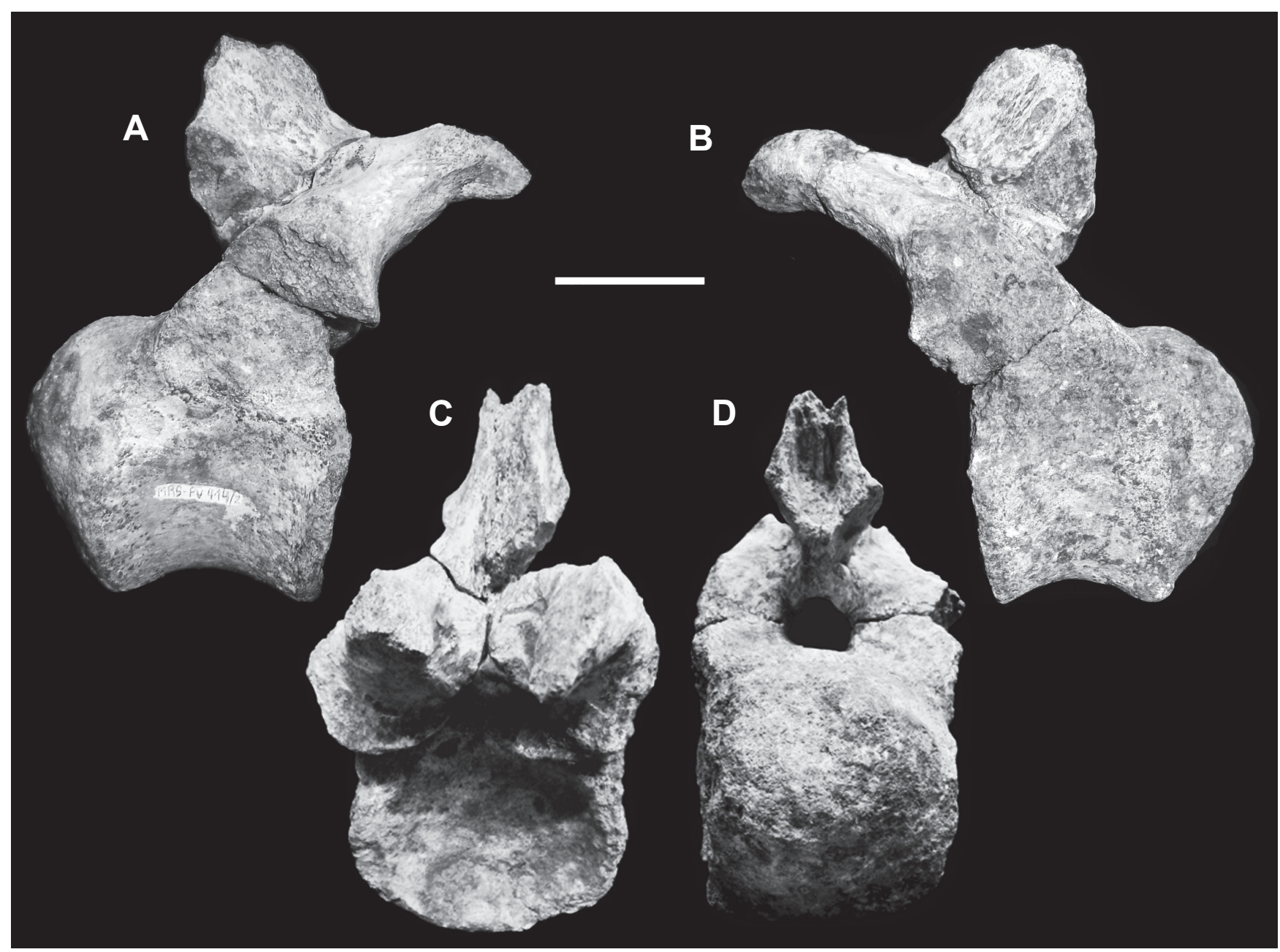

Figura 4. Aeolosaurini indet. MAU-Pv-N-414. Detalle de la vértebra caudal 6 en vistas lateral derecha (A), lateral izquierda (B), anterior (C) y posterior (D). Escala $=5 \mathrm{~cm}$.

Figure 4. Aeolosaurini indet. MAU-Pv-N-414, detail of the caudal vertebra 6 in right lateral (A), left lateral (B), anterior (C) and posterior (D) views. Scale bar $=5 \mathrm{~cm}$.

Rincosaurus caudamirus fue originalmente relacionado con Aeolosaurus, en un clado débilmente soportado (Nodo 11 de Calvo \& González Riga, 2003). Un análisis posterior posicionó a Rinconsaurus junto a Muyelensaurus pecheni, en el clado Rinconsauria (Calvo et al., 2007). Asimismo, Calvo et al. (2007) discutieron la ausencia en Rinconsaurus de numerosos rasgos típicos de aeolosaurinos y sustentaron fuertemente Rinconsauria. En el análisis de Santucci \& Arruda-Campos (2011), Rinconsaurus es posicionado en una politomía constituida por Maxakalisaurus Kellner et al., 2006, Panamericansaurus, Rincosaurus y el clado integrado por Gondwanatitan Kellner \& Azevedo, 1999, Aelosaurus maximus, A. rionegrinus y A. colhuehuapensis. En ese análisis, el clado Aeolosaurini está sólo soportado por un carácter (i.e. centros caudales posteriores dorsoventralmente bajos). De este modo, la posición basal de Rincosaurus dentro de Aeolosaurini no es conclusiva, teniendo en cuenta, asimismo, la hipótesis de Calvo et al. (2007).

Otros materiales asignados a Aeolosaurini, como los registros de Aeolosaurus sp. de las formaciones Allen y Los Alamitos, presentan una combinación de caracteres y variaciones morfológicas que dificultan su asignación específica (por ello considerados originalmente Aeolosaurus sp.) y con la descripción de nuevos géneros de Aeolosaurini, también se pone en duda su asignación genérica. Este conjunto de factores es, en parte, producto de la condición fragmentaria de muchos de los especímenes estudiados. Por ejemplo, la asignación al género Aeolosaurus de MPCA 27174 de la Formación Allen (Salgado \& Coria, 1993; García \& Salgado, en prensa) es aún tema de debate ya que posee las postzigapofisis retraídas respecto al borde anterior del cuerpo vertebral. Asimismo, este material fue recientemente referido a Panamericansaurus schroederi por Juárez Valieri \& Bellardini (2012).

Basados en la propuesta de Salgado \& Coria (1993, p. 126-127) para la determinación del largo relativo de las prezigapófisis y la posición relativa de las postzigapófisis en vértebras caudales medias, ampliamos los datos presentados originalmente por Salgado \& Coria (1993) con la inclusión de especímenes tradicionalmente incluidos en Aeolosaurini y algunos géneros externos (Figura 6, Tabla 1). Como resultado obtuvimos una proximidad entre el holotipo de Aeolosaurus rionegrinus y A. colhuehuapensis, y separadamente entre los 


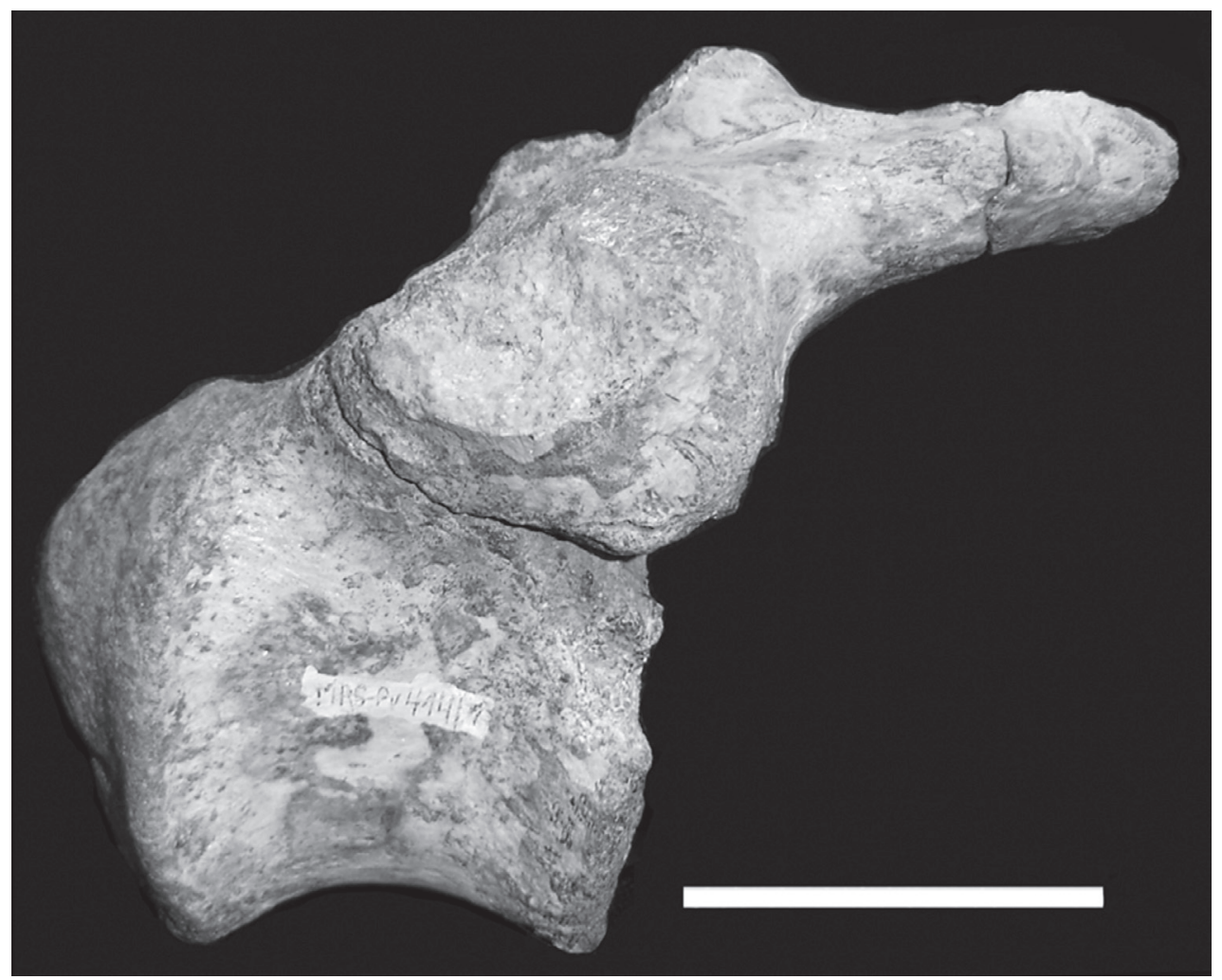

Figura 5. Aeolosaurini indet. MAU-Pv-N-414. Detalle de la vértebra caudal 7 en vista lateral derecha. Escala $=5 \mathrm{~cm}$.

Figure 5. Aeolosaurini indet. MAU-Pv-N-414, detail of the caudal vertebra 7 in right lateral view. Scale bar $=5 \mathrm{~cm}$.

Tabla 1. Datos utilizados para la realización de la Figura 5 donde se grafica la relación de medidas obtenidas para el largo relativo de la prezigapófisis y la posición relativa de la postzigapófisis en vértebras caudales medias de diversos titanosaurios, según esquema propuesto por Salgado \& Coria (1993, p. 127). Puntos utilizados para la medición de segmentos: A, extremo anterior de la cara articular de la prezigapófisis; B, borde antero-ventral del cuerpo vertebral; C, extremo anterior de la cara articular de la postzigapófisis; D, extremo posterior del cuerpo vertebral en el ápice del cóndilo articular (véase Figura 5).

Table 1. Data used for the realization of the Figure 5 where is plotted the relationship of measurement obtained for the relative long of the prezygapophysis and the relative position of the postzygapophysis in middle caudal vertebrae of some titanosaurs, according to the outline proposed by Salgado \& Coria (1993, p. 127). Points used to measurement segments: A, anterior point of the articular surface of the prezygapophysis; B, antero-ventral edge of the vertebral body; C, anterior point of the articular surface of the postzygapophysis; D, posterior point (maximum) of the vertebrate body at the convex articular surface (see Figura 5).

\begin{tabular}{|c|c|c|c|}
\hline Taxón o espécimen & $\mathrm{CD} / \mathrm{BD}$ & $\mathrm{AD} / \mathrm{BD}$ & Vértebra usada y bibliografía \\
\hline Baurutitan britoi & 0.53 & 1.73 & Caudal 6a; Kellner et al., 2005, fig. 16 \\
\hline Petrobrasaurus puestohernandezi & 0.74 & 1.67 & Caudal media-anterior; Filippi et al., 2011b, fig. 5G \\
\hline Aeolosaurus rionegrinus & 1.44 & 2.47 & 8ª Caudal; Powell, 2003, lám. 11, 2 \\
\hline Aeolosaurus colhuelhuapensis & 1.22 & 2.21 & Caudal 9a?; Casal et al., 2007, fig. 3C \\
\hline Aeolosaurus sp. MPCA 27174 & 0.95 & 1.91 & Caudal media; Salgado \& Coria, 1993, fig. 3 \\
\hline Aeolosaurus maximus & 0.84 & 1.82 & Caudal media anterior; Santucci \& Arruda-Campos, 2011, fig. 4J \\
\hline Panamericansaurus schroederi & 1.06 & 1.87 & Caudal anterior-media; Calvo \& Porfiri, 2010, fig. 3B \\
\hline Gondwanatitan faustoi & 0.84 & 1.97 & Caudal 5a; Kellner \& Azevedo, 1999, figs. 12-13 \\
\hline MAU-Pv-N-414 & 1.1 & 1.76 & Caudal $6^{\text {a?}}$; este trabajo \\
\hline
\end{tabular}

demás especímenes (Figura 6). Debido a la disponibilidad del material, un problema que enfrenta este gráfico es la selección de la vértebra caudal media que es usada para medición, ya que no es exactamente la misma en todos los ejemplares considerados. Baurutitan Kellner, Campos \& Trotta, 2005 y Petrobrasaurus, los dos taxones no Aeolosaurini (sensu Santucci \& Arruda-Campos 2011 y Filippi et al., 2011b) usados en el análisis, se posicionan fuera de la nube principal de Aeolosaurini no Aeolosaurus. Los especímenes de Aeolosaurus sp., A. maximus, Gondwanatitan, Panamericansaurus, y el material aquí presentado muestran una relación intermedia entre no Aeolosaurini (e.g. Baurutitan; sensu análisis filogenético de Santucci \& ArrudaCampos, 2011) y las especies patagónicas $A$. rionegrinus y A. colhuehuapensis, como fue resaltado por Salgado \& Coria (1993), para Aeolosaurus sp. de la Formación Allen. Este análisis muestra que el largo relativo de las prezigapófisis y la posición relativa de las postzigapófisis no es concluyente 


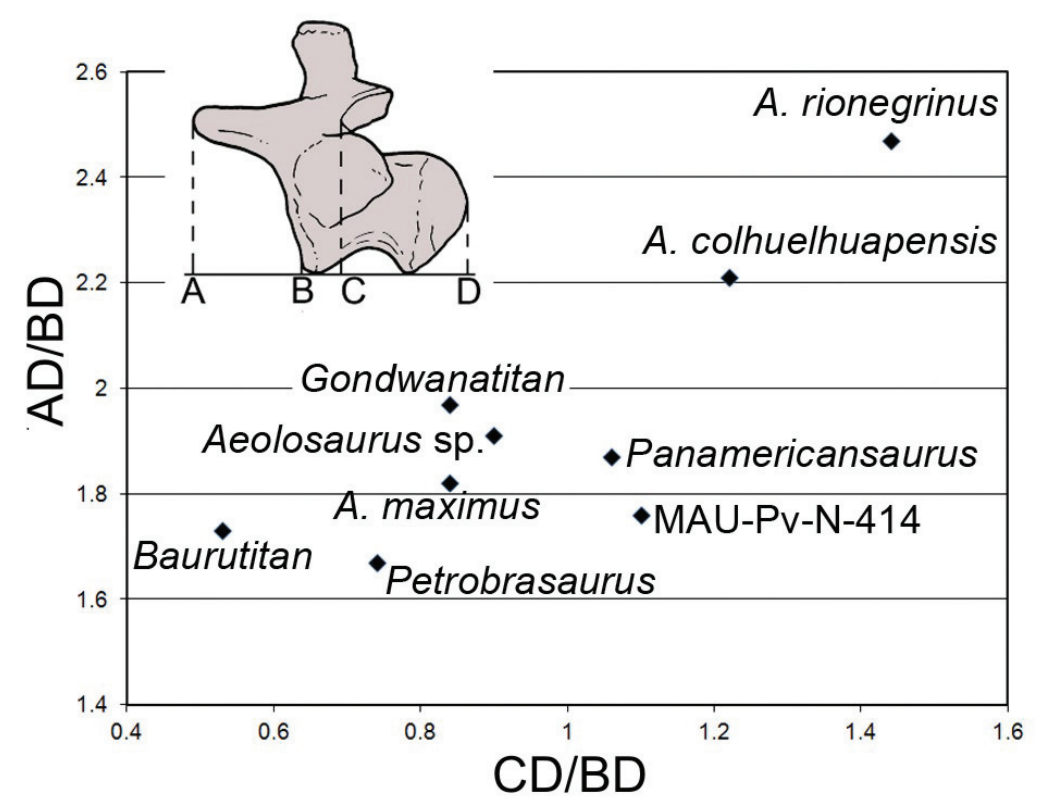

Figura 6. Determinación del largo relativo de las prezigapófisis y la posición relativa de las postzigapófisis en vértebras caudales medias de algunos titanosaurios sudamericanos (véase Tabla1).

Figure 6. Determination of the prezygapophyses relative length and the relative position of the postzigapophyses in middle caudal vertebrae of some South American titanosaurs (see Table1).

para distinguir taxones dentro de Aeolosaurini (posiblemente a excepción de A. rionegrinus y A. colhuehuapensis), y que el material holotipo de $A$. rionegrinus y A. colhuelhuapensis son los únicos que muestran una condición extrema, posiblemente sinapomórfica de este género. Por ello, un número mayor de caracteres serán necesarios para corroborar las asignaciones de los materiales de la Patagonia al género Aeolosaurus.

\section{El clado Aeolosaurini en Brasil}

Hasta la descripción de Aeolosaurus maximus del Cretácico Superior del Estado de São Paulo (Brasil), los registros brasileños de Aeolosaurus estaban basados en materiales sumamente escasos (e.g. Santucci \& Bertini, 2001; López \& Buchmann, 2008) o parcialmente descriptos, como fueron las primeras menciones del holotipo de $A$. maximus originalmente referenciado como "MPMA/sin número" proveniente de la localidad de Monte Alto, SP (Santucci \& Bertini, 2001). Las últimas revisiones realizadas sobre el género Aeolosaurus en Brasil fueron publicadas por Martinelli et al. (2011) y Santucci \& Arruda-Campos (2011). Martinelli et al. (2011) concluyen que hasta el momento no hay registros bien fundamentados del género Aeolosaurus en Brasil. Al momento de efectuarse esa publicación, el material holotipo de A. maximus estaba sólo parcialmente publicado por Santucci \& Bertini (2001), y el justificativo usado por Martinelli et al. (2011) para excluir este espécimen brasileño del género Aeolosaurus fue la posición relativa de las postzigapofisis en las vértebras caudales anteriores y medias. La posición anterior de las postzigapófisis, a nivel del borde anterior del cuerpo vertebral, fue un estado propuesto como autapomorfía del género Aeolosaurus por Casal et al. (2007) que no se encuentra presente en ninguna de las vértebras caudales del holotipo de Aeolosaurus maximus de Brasil.
Por el contrario, Santucci \& Arruda-Campos (2011) resaltan que la posición de las postzigapófisis varia dentro de una misma secuencia caudal. A pesar de ello, al menos una de las vertebras caudales de Aeolosaurus rionegrinus y A. colhuehuapensis poseen las postzigapófisis ubicadas anteriormente, prácticamente sobre el borde anterior del cuerpo vertebral (Powell, 2003; Casal et al., 2007), condición ausente en todos los Aeolosaurini de Brasil. En el análisis filogenético de Santucci \& Arruda-Campos (2011) el espécimen de Monte Alto se posiciona como grupo hermano de las especies patagónicas A. rionegrinus y A. colhuehuapensis. Este clado es soportado por dos caracteres, siendo la única sinapomorfía no ambigua la presencia de prezigapófisis curvadas ventralmente en las caudales más anteriores (Santucci \& Arruda-Campos, 2011, carácter 238, estado 1). Esta condición (i.e. curvatura ventral) es extrema en A. maximus (véase Santucci \& ArrudaCampos, 2011, fig. 4A) y, a lo que parece, poco acentuada o ausente en el holotipo de A. rionegrinus (véase Powell, 2003, plate 10, fig. 2a) y en A. colhuehuapensis (véase Casal et al., 2007, figs. 2,3). Como señalan Santucci \& Arruda-Campos (2011), las prezigapofisis del holotipo de A. rionegrinus son más similares a las del espécimen MPCA 27174, Aeolosaurus sp., de la Formación Allen (Salgado \& Coria, 1993), que a las de otros materiales referidos a Aeolosaurus. Con respecto al material aquí analizado, la vertebra C6 posee la prezigapófisis con el borde ventral suavemente cóncavo, aunque no tan acentuado como en A. maximus, y la faceta articular proyectada ventralmente, similar a la del género Aeolosaurus. Esta gran variabilidad en los procesos vertebrales de los arcos neurales de las vertebras caudales genera problemas al momento de reconocer afinidades, especialmente conociendo aún escasamente las variaciones inter- e intraespecíficas de la mayoría de los taxones. 
Finalmente, otro material que aporta controversias sobre su asignación es el espécimen CPPLIP 248 (vertebra caudal media, aislada) de la Formación Marília, localidad de Peirópolis (Uberaba, MG), originalmente mencionado por Santucci \& Bertini (2001) como Aeolosaurus sp. Martinelli et al. (2011) consideran a CPPLIP 248 como un Aeolosaurini indet. debido a la ausencia de caracteres autapomórficos del género Aeolosaurus (sensu Casal et al., 2007). Por otra parte, Santucci \& Arruda-Campos (2011, p. 22) resaltan la ausencia de caracteres diagnósticos del género Aeolosaurus, propuestos por ellos, en el espécimen CPPLIP 248. Sin embargo, los autores optan por asignar el espécimen CPPLIP 248 al género Aeolosaurus por la ausencia del contorno en forma de "corazón" de la superficie articular posterior del cuerpo vertebral, una característica presente en el Aeolosaurini Gondwanatitan. Se cree que debido a la ausencia de autapomorfias del género Aeolosaurus o de alguna de sus especies, es más oportuno considerar CPPLIP 248 como un Aeolosaurini indet. que como un Aeolosaurus sp. Asimismo, como ya fue mencionado por otros autores (e.g. Salgado \& García, 2002), el contorno en forma de corazón es un carácter que varia dentro de la serie caudal debido, presumiblemente, a la distribución de los paquetes del músculo caudofemoralis, por lo que debe ser usado con cautela. En Gondwanatitan, al menos desde la vertebra caudal $13^{\circ}$, el centro va adquiriendo un contorno cuadrangular posteriormente (Kellner \& Azevedo, 1999, p. 126). Debido a la ausencia de autapomorfias del género Aeolosaurus [sensu diagnosis de Casal et al. (2007) y Santucci \& ArrudaCampos (2011)] y a la condición fragmentaria del material, se cree que es más parsimonioso considerar CPP 248 como un Aeolosaurini indet.

\section{CONCLUSIONES}

El ejemplar MAU-Pv-N-414, basado en una secuencia caudal incompleta, es asignado al clado Aeolosaurini (Dinosauria, Sauropoda, Titanosauria). Su presencia en la Formación Plottier proporciona un nuevo elemento faunístico para esta unidad, y particularmente para Rincón de los Sauces (Provincia de Neuquén), en donde se ha registrado una diversidad de dinosaurios saurópodos, como MAU-Pv-AC-01 gen. et sp. indet., Rinconsaurus caudamirus, Muyelensaurus pecheni, Pitekunsaurus macayai, Narambuenatitan palomoi y Petrobrasaurus puestohernandezi. A pesar de la naturaleza fragmentaria de MAU-Pv-N-414, este ejemplar

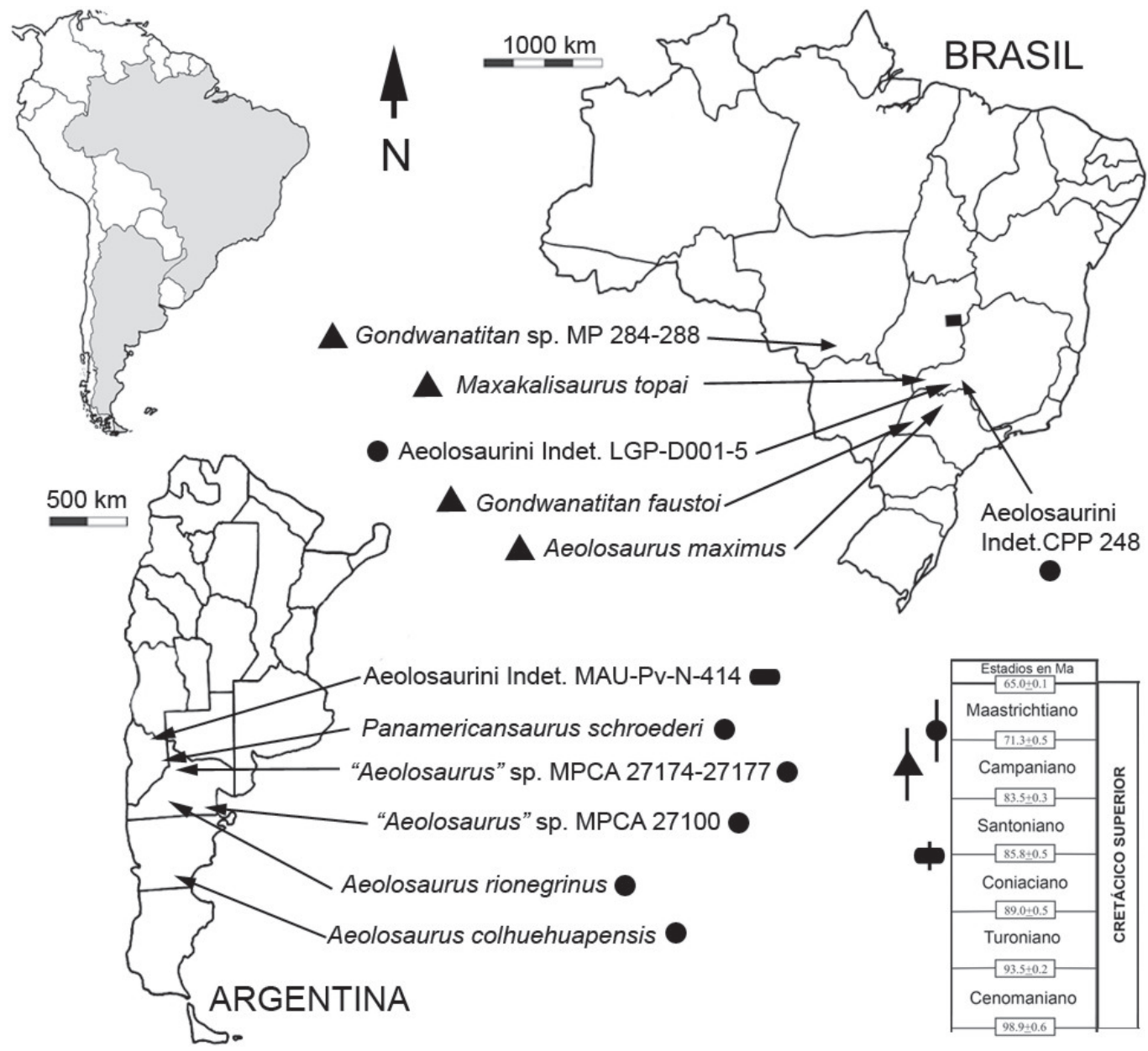

Figura 7. Registro de los Aeolosaurini en Brasil y en Argentina.

Figure 7. Record of the Aeolosaurini from Brazil and Argentina. 
es diferenciable de los restantes taxones reconocidos en el norte de Neuquén. Asimismo, este espécimen constituye el registro más antiguo del clado Aeolosaurini (Figura 7). La taxonomía y relaciones filogenéticas de los Aeolosaurini son aún controvertidas, y nuevos y exhaustivos análisis serán necesarios para la resolución de esta problemática.

\section{AGRADECIMIENTOS}

Los autores agradecen a S. Palomo por indicar el sitio del hallazgo y por la preparación del material, a L.C.B Ribeiro (CPP-CCCP/UFTM) y V.P.A. Teixeira (CPP-CCCP/UFTM) por el acceso a las colecciones a su cargo. AGM fue beneficiado con una beca $\mathrm{CNPq} /$ Brasil bajo la dirección de V.P.A. Teixeira (CCCP/UFTM). Finalmente un agradecimiento a L. Salgado y a un revisor anónimo por los aportes realizados que mejoraron significativamente el manuscrito.

\section{REFERENCIAS}

Calvo, J.O; Coria, R.A. \& Salgado, L. 1997. Uno de los más completos titanosáuridos (Dinosauria-Sauropoda) registrados en el mundo. Ameghiniana, 34:534.

Calvo, J.O. \& González Riga, B.J. 2003. Rinconsaurus caudamirus gen. et sp. nov., a new titanosaurid (Dinosauria, Sauropoda) from the Late Cretaceous of Patagonia, Argentina. Revista Geológica de Chile, 2:333-353.

Calvo, J.O.; González Riga, B.J. \& Porfiri, J.D. 2007. A new titanosaur sauropod from the Late Cretaceous of Neuquén, Patagonia, Argentina. Arquivos do Museu Nacional, 4:485-504.

Calvo, J.O. \& Porfiri, J.D. 2010. Panamericansaurus schroederi gen. nov. sp. nov. un nuevo Sauropoda (Titanosauridae-Aeolosaurini) de la Provincia del Neuquén, Cretácico Superior de Patagonia, Argentina. Brazilian Geographical Journal: Geosciences and Humanities research medium, 1:100-115.

Campos, D.A.; Kellner, A.W.A.; Bertini, R.J. \& Santucci, R.M. 2005. On a titanosaur (Dinosauria, Sauropoda, Titanosauridae) vertebral column from the Bauru Group, Late Cretaceous of Brazil. Arquivos do Museu Nacional, 63:565-593.

Casal, G.; Martínez, R.; Luna, M.; Sciutto, J.C. \& Lamanna, M. 2007. Aeolosaurus colhuehuapensis sp. nov. (Sauropoda, Titanosauria) de la Formación Bajo Barreal, Cretácico Superior de Argentina. Revista Brasilera de Paleontologia, 10:53-62.

Filippi, L.S.; Canudo, J. I.; Salgado, L.; Garrido, A.; García, R.; Cerda, I.A. \& Otero, A. 2011b. A new sauropod: Petrobrasaurus puestohernandezi gen et sp. nov. from the Upper Cretaceous (Plottier Formation) of Rincón de los Sauces, Neuquén (Argentina). Geologica Acta, 9:1-12.doi:10.1344/105.000001648

Filippi, L.S.; García, R.A. \& Garrido, A.C. 2011a. A new titanosaur sauropod dinosaur from Upper Cretaceous of North Patagonia, Argentina. Acta Paleontológica Polonica, 56:505-520. doi:10.4202/ app.2010.0019

Filippi,L.S. \& Garrido.A.C. 2008. Pitekunsaurus macayai gen. et sp. nov., nuevo titanosaurio (Saurischia, Sauropoda) del Cretácico Superior de la Cuenca Neuquina, Argentina. Ameghiniana, 45:575-590.

Franco-Rosas, C.A.; Salgado, L.; Rosas, C.F. \& Carvalho, I.S. 2004. Nuevos materiales de titanosaurios (Saurópoda) en el Cretácico Superior de Mato Grosso, Brasil. Revista Brasileira de Paleontologia, 7:329-336.
Garcia, R.A. \& Salgado, L. (en prensa). The titanosaur sauropods from the Allen Formation (late Campanian-early Maastrichtian) of Salitral Moreno (Patagonia, Río Negro, Argentina). Acta Palaeontologica Polonica, doi:10.4202/app.2011

Garrido, A.C. 2010. Estratigrafía del Grupo Neuquén, Cretácico Superior de la Cuenca Neuquina (Argentina): nueva propuesta de ordenamiento litoestratigráfico. Revista del Museo Argentino de Ciencias Naturales, 12:121-177.

Juárez Valieri, R.D. \& Bellardini, F. 2012. Los Aeolosaurini (Sauropoda, Titanosauria) de la Formación Allen, implicaciones taxonómicas y bioestratigráficas. In: JORNADAS ARGENTINAS DE PALEONTOLOGÍA DE VERTEBRADOS, 26, 2012. Resúmenes, Buenos Aires, Universidad Maimónides, CDROM.

Kellner, A.W.A. \& Azevedo, A.K. 1999. A new sauropod dinosaur (Titanosauria) from the Late Cretaceous of Brazil. In: Y. Tomida, T.H. Rich \& P. Vickers-Rich (eds.) Proceedings of the second Gondwanan Dinosaur Symposium, Tokyo, p. 111-142 (National Science Museum Monograph 15).

Kellner, A.W.A.; Campos, D. A.; Azevedo, S.A.K.; Trotta, M.N.F; Henriques, D.D.R.; Craik, M.M.T. \& De Paula Silva, H. 2006. On a new Titanosaur sauropod from the Bauru Group, Late Cretaceous of Brazil. Boletim do Museu Nacional, 74:1-31.

Kellner, A.W.A.; Campos, D.A. \& Trotta, M.N. 2005. Description of a titanosaurid caudal series from the Bauru Group, late Cretaceous of Brazil. Arquivos do Museu Nacional, 63:529-564.

Lopes, R.P. \& Buchmann, F.S.C. 2008. Fossils of titanosaurs (Dinosauria, Sauropoda) from a new outcrop in Triângulo Mineiro, southeastern Brazil. Revista Brasilera de Paleontologia, 11:69-72. doi.org/10.4072/rbp.2008.1.07

Martinelli, A.G.; Riff, D. \& Lopes, R.P. 2011. Discussion about the occurrence of the genus Aeolosaurus Powell 1987 (Dinosauria, Titanosauria) in the Upper Cretaceous of Brazil. Gaea, Journal of Geociences, 7:34-40. doi: 10.4013/gaea.2011.71.03

Powell, J.E. 1987. The Late Cretaceous fauna of Los Alamitos, Patagonia, Argentina. Part VI. The titanosaurids. Revista del Museo Argentino de Ciencias Naturales "Bernardino Rivadavia", 3:147-153.

Powell, J.E. 2003. Revision of South American Titanosaurid dinosaur: palaeobiological, palaeobiogeoraphical and phylogenetic aspects. Records of the Queen Victoria Museum, 111:1-173.

Ramos, V.A. 1981. Descripción Geológica de la Hoja 33c, Los Chihuidos Norte. Provincia del Neuquén. Boletín Servicio Geológico Nacional, 182:1-103.

Salgado, L. \& Coria, R.A. 1993. El género Aeolosaurus (Sauropoda. Titanosauridae) en la Formación Allen (CampanianoMaastrichtiano) de la Provincia de Río Negro, Argentina. Ameghiniana, 30:119-128.

Salgado, L.; Coria, R.A. \& Calvo, J.O. 1997. Presencia del género Aeolosaurus (Sauropoda, Titanosauridae) en la Formación Los Alamitos, Cretácico Superior de la Provincia de Río Negro, Argentina. Revista Universidade Guarulhos, 2:44-49.

Santucci, R.M. \& Arruda-Campos, A. C.2011. A new sauropod (Macronaria, Titanosauria) from the Adamantina Formation, Bauru Group, Upper Cretaceous of Brazil and the phylogenetic relationships of Aeolosaurini. Zootaxa, 3085:1-33.

Santucci, R.M. \& Bertini, R.J. 2001. Distribuição paleogeográfica e biocronológica dos titanossauros (Saurischia, Sauropoda) do Grupo Bauru, Cretáceo Superior do sudeste brasileiro. Revista Brasileira de Geociências, 31:307-314.

Received in September, 2012; accepted in March, 2013. 